\title{
Ground State Solutions for a Kind of Schrödinger-Poisson System with Upper Critical Exponential Convolution Term
}

\author{
Yaolan Tang, Qiongfen Zhang* \\ College of Science, Guilin University of Technology, Guilin, China \\ Email: *qfzhangcsu@163.com
}

How to cite this paper: Tang, Y.L. and Zhang, Q.F. (2022) Ground State Solutions for a Kind of Schrödinger-Poisson System with Upper Critical Exponential Convolution Term. Journal of Applied Mathematics and Physics, 10, 576-588.

https://doi.org/10.4236/jamp.2022.102042

Received: January 18, 2021

Accepted: February 22, 2022

Published: February 25, 2022

Copyright (c) 2022 by author(s) and Scientific Research Publishing Inc. This work is licensed under the Creative Commons Attribution International License (CC BY 4.0).

http://creativecommons.org/licenses/by/4.0/

\begin{abstract}
This paper mainly discusses the following equation:

$\left\{\begin{array}{ll}-\Delta u+V(x) u+\lambda \phi u=\left(I_{\alpha} *|u|^{3+\alpha}\right)|u|^{1+\alpha} u, & x \in \mathbb{R}^{3}, \\ -\Delta \phi=u^{2}, & x \in \mathbb{R}^{3},\end{array}\right.$ where the potential function $V: \mathbb{R}^{3} \rightarrow \mathbb{R}, \quad \alpha \in(0,3), \lambda>0$ is a parameter and $I_{\alpha}$ is the Riesz potential. We study a class of Schrödinger-Poisson system with convolution term for upper critical exponent. By using some new tricks and NehairPohožave manifold which is presented to overcome the difficulties due to the presence of upper critical exponential convolution term, we prove that the above problem admits a ground state solution.
\end{abstract}

\section{Keywords}

Convolution Nonlinearity, Schrödinger-Poisson System, Upper Critical Exponent, Ground State Solution

\section{Introduction}

Recently, the following Schrödinger-Poisson system has been studied widely by researchers

$$
\begin{cases}-\Delta u+V(x) u+\lambda \phi u=f(u), & x \in \mathbb{R}^{N} ; \\ -\Delta \phi=u^{2}, & x \in \mathbb{R}^{N},\end{cases}
$$

where $\lambda>0, N \geq 3$, the external potential function $V \in \mathcal{C}\left(\mathbb{R}^{3}, \mathbb{R}\right)$ and the nonlinearity $f \in \mathcal{C}\left(\mathbb{R}^{3} \times \mathbb{R}, \mathbb{R}\right) .(1.1)$ is also called Schrödinger-Maxwell system, which appears in an amusing physical background. In fact, based on a classical physical model, coupled nonlinear Schrödinger-Poisson equation can be used to 
describe the interaction between charge particles and electromagnetic field. For more physical contexts of the Schrödinger-Poisson system, we refer the readers to the papers [1] [2] and the references therein.

There are lots of extended research on (1.1) in $\mathbb{R}^{3}$. When $V(x) \equiv V>0$ is a constant and $N=3$, Khoutir [3] proved that (1.1) possesses a least energy sign-changing solution and a ground state solution by variational methods under some relaxed assumptions on $f$. When $\lambda=1,(1.1)$ reduces to the following class of Schrödinger-Possion system

$$
\begin{cases}-\Delta u+V(x) u+\phi u=f(u), & x \in \mathbb{R}^{N} ; \\ -\Delta \phi=u^{2}, & x \in \mathbb{R}^{N},\end{cases}
$$

when $V(x)=1$, by introducing some new variational and analytic techniques, Chen, Shi and Tang [4] showed that (1.2) has a nontrivial solution of mountain pass type and a ground state solution of Neheri-Pohožaev type in $\mathbb{R}^{2}$. By variational methods and Miranda's theorem, Alves et al. [5] proved that (1.2) admits a least energy sign-changing solution in $\mathbb{R}^{3}$ when $f$ satisfies some special assumptions. Similarly, combining constraint variational method and quantitative deformation lemma, Shuai and Wang [6] proved that (1.1) possesses a sign-changing solution $u_{\lambda}$. Moreover, they showed that any sign-changing solution of (1.1) has energy exceeding more than twice the least energy. There are a lot of works about (1.2) and we refer to the literature [6] [7] and references therein.

Without the internal potential $\phi u,(1.1)$ reduces to the following Schrödinger equation:

$$
\left\{\begin{array}{l}
-\Delta u+V(x) u=f(u), \quad x \in \mathbb{R}^{N} \\
u \in H^{1}\left(\mathbb{R}^{N}\right) .
\end{array}\right.
$$

Using Berestycki-Lions conditions on $f$, Chen and Tang [8] studied generalized nonlinear Schrödinger equation with variable potential. By introducing skillful ideas and relaxed assumptions on $V(x)$, they obtain a ground state solution of Pohožaev type and a least energy solution. Besides, there are many results of sign-changing ground state solutions of (1.3). We refer to [9]-[15] and references therein.

Let $\lambda=1$ and $f(u)=\left(I_{\alpha} * G(u)\right) g(u)$, the Schrödinger-Poisson system (1.1) becomes the following equation with convolution nonlinearity.

$$
\begin{cases}-\Delta u+V(x) u+\phi u=\left(I_{\alpha} * G(u)\right) g(u), & x \in \mathbb{R}^{3} ; \\ -\Delta \phi=u^{2}, & x \in \mathbb{R}^{3},\end{cases}
$$

where $\alpha \in(0,3), g \in \mathcal{C}(\mathbb{R}, \mathbb{R})$ and $G(u)=\int_{0}^{t} g(s) \mathrm{d} s$. Under mild assumptions on nonlinear perturbation $g$ and $V$, Chen and Tang [11] proved that (1.4) has a ground state solution in two cases by using new inequalities. In their work, when $0<\alpha<2$, they established the Nehari-Pohožaev manifold and proved that (1.4) has a solution. Next, they defined the Nehari manifold to obtain the existence of the solution when $2 \leq \alpha<3$. For more details about assumptions and techniques of (1.4), we refer to [6] [11] [16] [17] [18]. 
In this paper, we mainly focus on the following equations:

$$
\begin{cases}-\Delta u+V(x) u+\lambda \phi u=\left(I_{\alpha} *|u|^{3+\alpha}\right)|u|^{1+\alpha} u, & x \in \mathbb{R}^{3} ; \\ -\Delta \phi=u^{2}, & x \in \mathbb{R}^{3},\end{cases}
$$

where $V$ satisfies the following assumptions:

(V1) $V \in \mathcal{C}\left(\mathbb{R}^{3},[0, \infty)\right), V(x) \leq V_{\infty}:=\lim _{|x| \rightarrow \infty} V(x)$ for all $x \in \mathbb{R}^{3}$ and $V_{\infty}>0$;

(V2) $V \in \mathcal{C}\left(\mathbb{R}^{3}\right)$, the set $\left\{x \in \mathbb{R}^{3}:|\nabla V(x) \cdot x| \geq \varepsilon\right\}$ has finite Lebesgue measure for every $\varepsilon>0$, and the function $t \mapsto t^{2}[V(t x)-\nabla V(t x) \cdot(t x)]$ is increasing on $(0, \infty)$ for every $x \in \mathbb{R}^{3}$.

In three-dimensional space, the Riesz potential $I_{\alpha}$ is defined as a function of $\mathbb{R}^{3} \rightarrow \mathbb{R}:$

$$
I_{\alpha}(x)=\frac{\Gamma\left(\frac{3-\alpha}{2}\right)}{\Gamma\left(\frac{\alpha}{2}\right) 2^{\alpha} \pi^{3 / 2}|x|^{3-\alpha}}, \quad x \in \mathbb{R}^{3} \backslash\{0\},
$$

where $\Gamma(\cdot)$ is the Gamma function. It is widely known that for any $u \in H^{1}\left(\mathbb{R}^{3}\right)$, there exists a unique $\phi_{u} \in \mathcal{D}^{1,2}\left(\mathbb{R}^{3}\right)$ such that $-\Delta \phi=u^{2}$ by using the LaxMilgram theorem, moreover,

$$
\phi_{u}(x)=\int_{\mathbb{R}^{3}} \frac{u^{2}(y)}{|x-y|} \mathrm{d} y=\frac{1}{|x|} * u^{2} .
$$

Inserting (1.6) into (1.5), we get the following equation

$$
-\Delta u+V(x) u+\phi_{u}(x) u=\left(I_{\alpha} *|u|^{3+\alpha}\right)|u|^{1+\alpha} u .
$$

The following inequality, which is a special case of Hardy-Littlewood-Sobolev inequality, plays a significant role in resolving the difficulty of relatively compact. There exists sharp constant $S$, independent of $u$, such that

$$
\left[\int_{\mathbb{R}^{3}}\left(I_{\alpha} *|u|^{3+\alpha}\right)|u|^{3+\alpha} \mathrm{d} x\right]^{\frac{1}{3+\alpha}} \leq S^{-1} \int_{\mathbb{R}^{3}}|\nabla u|^{2} \mathrm{~d} x,
$$

whose external function is

$$
u(x)=C\left(\frac{\lambda_{1}}{\lambda_{1}^{2}+|x|^{2}}\right)^{\frac{1}{2}},
$$

$\int_{\mathbb{R}^{3}}\left(I_{\alpha} *|u|^{3+\alpha}\right)|u|^{3+\alpha} \mathrm{d} x$ is invariant under dilations $\lambda_{1}^{\frac{1}{2}} u(\lambda)$ [19]. Next, we define the energy functional:

$$
\begin{aligned}
E(u)= & \frac{1}{2} \int_{\mathbb{R}^{3}}\left[|\nabla u|^{2}+V(x) u^{2}\right] \mathrm{d} x+\frac{\lambda}{4} \int_{\mathbb{R}^{3}} \phi_{u}(x) u^{2} \mathrm{~d} x \\
& -\frac{1}{2(3+\alpha)} \int_{\mathbb{R}^{3}}\left(I_{\alpha} *|u|^{3+\alpha}\right)|u|^{3+\alpha} \mathrm{d} x .
\end{aligned}
$$


Then for any $u, v \in H^{1}\left(\mathbb{R}^{3}\right)$,

$$
\begin{aligned}
\left\langle E^{\prime}(u), v\right\rangle= & \int_{\mathbb{R}^{3}}[\nabla u \cdot \nabla v+V(x) u v] \mathrm{d} x+\lambda \int_{\mathbb{R}^{3}} \phi_{u}(x) u v \mathrm{~d} x \\
& -\int_{\mathbb{R}^{3}}\left(I_{\alpha} *|u|^{3+\alpha}\right)|u|^{2+\alpha} v \mathrm{~d} x .
\end{aligned}
$$

To state our result, we define the Nehari-Pohožaev manifold as follows:

$$
\mathcal{M}:=\left\{u \in H^{1}\left(\mathbb{R}^{3}\right) \backslash\{0\}: J(u):=2\left\langle E^{\prime}(u), u\right\rangle-\mathcal{P}(u)=0\right\}
$$

where

$$
\begin{aligned}
\mathcal{P}(u)= & \frac{1}{2}\|\nabla u\|_{2}^{2}+\frac{1}{2} \int_{\mathbb{R}^{3}}[3 V(x)+\nabla V(x) \cdot x] u^{2} \mathrm{~d} x+\frac{5 \lambda}{4} \int_{\mathbb{R}^{3}} \phi_{u} u^{2} \mathrm{~d} x \\
& -\frac{1}{2} \int_{\mathbb{R}^{3}}\left(I_{\alpha} *|u|^{3+\alpha}\right)|u|^{3+\alpha} \mathrm{d} x .
\end{aligned}
$$

Our main result is as follows.

Theorem 1.1. Assume $0<\alpha<3, V$ satisfies (V1), (V2). Then problem (1.5) has a ground state solution $\bar{u} \in H^{1}\left(\mathbb{R}^{3}\right)$ such that

$$
E(\bar{u})=\inf _{\mathcal{M}} E=\inf _{u \in H^{1}\left(\mathbb{R}^{3}\right) \backslash\{0\}} \max _{t>0} E\left(t^{2} u_{t}\right)>0 .
$$

\section{Notations.}

- $H^{1}\left(\mathbb{R}^{3}\right)$ denotes the usual Sobolev space equipped with the inner product and norm

$$
(u, v)=\int_{\mathbb{R}^{3}}(\nabla u \cdot \nabla v+u v) \mathrm{d} x, \quad\|u\|=(u, u)^{1 / 2}, \quad \forall u, v \in H^{1}\left(\mathbb{R}^{3}\right) .
$$

- $L^{s}\left(\mathbb{R}^{3}\right)(1<s<\infty)$ denotes the Lebesgue space with the norm

$$
\|u\|_{s}=\left(\int_{\mathbb{R}^{3}}|u|^{s} \mathrm{~d} x\right)^{1 / s} \text {. }
$$

- For any $u \in H^{1}\left(\mathbb{R}^{3}\right)$ and $r>0, B_{r}(x):=\{y \in \mathbb{R}:|y-x|<r\}$.

- For any $u \in H^{1}\left(\mathbb{R}^{3}\right) \backslash\{0\}, u_{t}(x):=u(t x)$ for $t>0$.

- $C_{1}, C_{2}, C_{3}, \cdots$ denote positive constants possibly different in different places.

\section{Preliminaries}

As usual, we assume $0<\alpha<3$. By (1.11) and (1.13), we have

$$
\begin{aligned}
J(u)= & \frac{3}{2}\|\nabla u\|_{2}^{2}+\frac{1}{2} \int_{\mathbb{R}^{3}}[V(x)-\nabla V(x) \cdot x] u^{2} \mathrm{~d} x+\frac{3 \lambda}{4} \int_{\mathbb{R}^{3}} \phi_{u} u^{2} \mathrm{~d} x \\
& -\frac{3}{2} \int_{\mathbb{R}^{3}}\left(I_{\alpha} *|u|^{3+\alpha}\right)|u|^{3+\alpha} \mathrm{d} x .
\end{aligned}
$$

First, we give some key inequalities.

$$
\begin{gathered}
f(x, t):=3[V(x)-t V(x / t)]-\left(1-t^{3}\right)[V(x)-\nabla V(x) \cdot x] \geq 0, \quad \forall x \in \mathbb{R}^{3}, t>0, \\
g(t):=\alpha+2-(3+\alpha) t^{3}+t^{9+3 \alpha}>0, \quad \forall t \in[0,1) \cup(1,+\infty) .
\end{gathered}
$$

Inspired by Tang and Chen [11], we establish a key functional inequality as follows. 
Lemma 2.1. Assunme that (V1) and (V2) hold. Then

$$
E(u) \geq E\left(t^{2} u_{t}\right)+\frac{1-t^{3}}{3} J(u)+\frac{1}{6} \int_{\mathbb{R}^{3}} f(x, t) u^{2} \mathrm{~d} x
$$

Proof. Note that

$$
\begin{aligned}
E\left(t^{2} u_{t}\right)= & \frac{t^{3}}{2} \int_{\mathbb{R}^{3}}|\nabla u|^{2} \mathrm{~d} x+\frac{t}{2} \int_{\mathbb{R}^{3}} V\left(t^{-1} x\right) u^{2} \mathrm{~d} x+\frac{\lambda t^{3}}{4} \int_{\mathbb{R}^{3}} \phi_{u}(x) u^{2} \mathrm{~d} x \\
& -\frac{t^{9+3 \alpha}}{2(3+\alpha)} \int_{\mathbb{R}^{3}}\left(I_{\alpha} *|u|^{3+\alpha}\right)|u|^{3+\alpha} \mathrm{d} x .
\end{aligned}
$$

Thus, by (1.10), (1.11), (2.2), (2.3) and (2.5), one has

$$
\begin{aligned}
& E(u)-E\left(t^{2} u_{t}\right) \\
&=\frac{1-t^{3}}{2} \|\left.\nabla u\right|_{2} ^{2}+\frac{1}{2} \int_{\mathbb{R}^{3}}\left[V(x)-t V\left(t^{-1} x\right)\right] u^{2} \mathrm{~d} x+\frac{\lambda\left(1-t^{3}\right)}{4} \int_{\mathbb{R}^{3}} \phi_{u}(x) u \mathrm{~d} x \\
&-\frac{1-t^{9+3 \alpha}}{2(3+\alpha)} \int_{\mathbb{R}^{3}}\left(I_{\alpha} *|u|^{3+\alpha}\right)|u|^{3+\alpha} \mathrm{d} x \\
&= \frac{1-t^{3}}{3} J(u)+\frac{1}{6} \int_{\mathbb{R}^{3}}\left\{3\left[V(x)-t V\left(t^{-1} x\right)\right]-\left(1-t^{3}\right)[V(x)-\nabla V(x) \cdot x]\right\} u^{2} \mathrm{~d} x \\
&+\frac{g(t)}{2(3+\alpha)} \int_{\mathbb{R}^{3}}\left(I_{\alpha} *|u|^{3+\alpha}\right)|u|^{3+\alpha} \mathrm{d} x \\
& \geq \frac{1-t^{3}}{3} J(u)+\frac{1}{6} \int_{\mathbb{R}^{3}} f(x, t) u^{2} \mathrm{~d} x .
\end{aligned}
$$

The proof of Lemma 2.1 is complete.

Assume that $t \rightarrow 0$, from (2.4), we have

$$
E(u) \geq \frac{1}{3} J(u)+\frac{1}{6} \int_{\mathbb{R}^{3}}[2 V(x)+\nabla V(x) \cdot x] u^{2} \mathrm{~d} x, \quad \forall u \in H^{1}\left(\mathbb{R}^{3}\right) .
$$

To solve the trouble caused by the lack of compactness of Sobolev space embedding in $\mathbb{R}^{3}$, we define the following energy functional when $V(x) \equiv V_{\infty}$

$$
\begin{aligned}
E^{\infty}(u)= & \frac{1}{2} \int_{\mathbb{R}^{3}}\left[|\nabla u|^{2}+V_{\infty} u^{2}\right] \mathrm{d} x+\frac{1}{4} \int_{\mathbb{R}^{3}} \lambda \phi_{u}(x) u^{2} \mathrm{~d} x \\
& -\frac{1}{2(3+\alpha)} \int_{\mathbb{R}^{3}}\left(I_{\alpha} *|u|^{3+\alpha}\right)|u|^{3+\alpha} \mathrm{d} x .
\end{aligned}
$$

According to (1.12) and (2.1), we define

$$
\mathcal{M}^{\infty}:=\left\{u \in H^{1}\left(\mathbb{R}^{3}\right) \backslash\{0\}: J^{\infty}(u)=0\right\},
$$

and

$$
J^{\infty}(u)=\frac{3}{2}\|\nabla u\|_{2}^{2}+\frac{V_{\infty}}{2}\|u\|_{2}^{2}+\frac{3 \lambda}{4} \int_{\mathbb{R}^{3}} \phi_{u} u^{2} \mathrm{~d} x-\frac{3}{2} \int_{\mathbb{R}^{3}}\left(I_{\alpha} *|u|^{3+\alpha}\right)|u|^{3+\alpha} \mathrm{d} x
$$

From Lemma 2.1, we can deduce the following corollaries.

Corollary 2.2. Assume that (V1) holds. Then 
$E^{\infty}(u) \geq E^{\infty}\left(t^{2} u_{t}\right)+\frac{1-t^{3}}{3} J^{\infty}(u)+\frac{(1-t)^{2}(2+t) V_{\infty}}{6}\|u\|_{2}^{2}, \quad \forall u \in H^{1}\left(\mathbb{R}^{3}\right), t \geq 0$

Corollary 2.3. Assume that (V1) and (V2) hold. Then for $u \in \mathcal{M}$

$$
E(u)=\max _{t>0} E\left(t^{2} u_{t}\right) .
$$

Lemma 2.4. ([11]: Lemma 2.7) Assume that (V1) and (V2) hold. Then there exist $\rho_{1}, \rho_{2}>0$ such that

$$
\begin{array}{cc}
2 V(x)+\nabla V(x) \cdot x \geq \rho_{1}, & \forall x \in \mathbb{R}^{3}, \\
V(x)-\nabla V(x) \cdot x \geq \rho_{2} & \forall x \in \mathbb{R}^{3} .
\end{array}
$$

Lemma 2.5. For any $u \in H^{1}\left(\mathbb{R}^{3}\right) \backslash\{0\}$, there exists a unique $t_{u}>0$ such that $t_{u}^{2} u_{t_{u}} \in \mathcal{M}$.

Proof. Let $u \in H^{1}\left(\mathbb{R}^{3}\right) \backslash\{0\}$ be fixed and define a function $\varsigma(t):=E\left(t^{2} u_{t}\right)$ on $(0, \infty)$. Clearly, by $(2.1)$ and $(2.5)$, we have

$$
\begin{gathered}
\varsigma^{\prime}(t)=0 \Leftrightarrow \frac{1}{2} \int_{\mathbb{R}^{3}}\left\{3 t^{3}|\nabla u|^{2}+t\left[V\left(t^{-1} x\right)-\nabla V\left(t^{-1} x\right) \cdot\left(t^{-1} x\right)\right] u^{2}\right\} \mathrm{d} x \\
+\frac{3 \lambda t^{3}}{4} \int_{\mathbb{R}^{3}} \phi_{u}(x) u^{2} \mathrm{~d} x-\frac{3 t^{9+3 \alpha}}{2} \int_{\mathbb{R}^{3}}\left(I_{\alpha} *|u|^{3+\alpha}\right)|u|^{3+\alpha} \mathrm{d} x=0 \\
\Leftrightarrow J\left(t^{2} u_{t}\right)=0 \Leftrightarrow t^{2} u_{t} \in \mathcal{M} .
\end{gathered}
$$

By (V1), one has $\varsigma(0)=0$ and $\varsigma(t)>0$ for $t>0$ small and $\varsigma(t)<0$ for $t$ large. Therefore, $\varsigma(t)$ has a critical point which corresponds to its maximum, namely, there is a $t_{0}=t_{u}>0$ so that $\varsigma^{\prime}\left(t_{0}\right)=0$ and $t_{0}^{2} u_{t_{0}} \in \mathcal{M}$. Then, we claim that $t_{u}$ is unique. Similar to the proof of ([20]: Lemma 3.3), for any $u \in H^{1}\left(\mathbb{R}^{3}\right) \backslash\{0\}$ which is given, if there are two positive constants $t_{1} \neq t_{2}$ such that $t_{1}^{2} u_{t_{1}}, t_{2}^{2} u_{t_{2}} \in \mathcal{M}$. Then $J\left(t_{1}^{2} u_{t_{1}}\right)=J\left(t_{2}^{2} u_{t_{2}}\right)=0$. Together with (2.4), we have

$$
\begin{aligned}
E\left(t_{1}^{2} u_{t_{1}}\right) & \geq E\left(t_{2}^{2} u_{t_{2}}\right)+\frac{t_{1}^{3}-t_{2}^{3}}{3 t_{1}^{3}} J\left(t_{1}^{2} u_{t_{1}}\right)+\frac{t_{1}}{6} \int_{\mathbb{R}^{3}} f\left(x, t_{2} / t_{1}\right) u^{2} \mathrm{~d} x \\
& \geq E\left(t_{2}^{2} u_{t_{2}}\right)+\frac{t_{1} f\left(x, t_{2} / t_{1}\right)}{6}\|u\|_{2}^{2},
\end{aligned}
$$

and

$$
E\left(t_{2}^{2} u_{t_{2}}\right) \geq E\left(t_{1}^{2} u_{t_{1}}\right)+\frac{t_{2} f\left(x, t_{1} / t_{2}\right)}{6}\|u\|_{2}^{2} .
$$

(2.2), (2.16) and (2.17) imply $t_{1}=t_{2}$. Hence, $t_{u}>0$ is unique for any $u \in H^{1}\left(\mathbb{R}^{3}\right) \backslash\{0\}$.

Corollary 2.6. For any $u \in H^{1}\left(\mathbb{R}^{3}\right) \backslash\{0\}$, there exists a unique $t_{u}>0$ such that $t_{u}^{2} u_{t_{u}} \in \mathcal{M}^{\infty}$.

Combing Corollary 2.3 and Lemma 2.5, we get $\mathcal{M}=\varnothing$ and the following minimax characterization.

Lemma 2.7. ([11]: Lemma 2.10) Assume (V1) and(V2) hold. Then 


$$
\inf _{u \in \mathcal{M}} E(u)=m=\inf _{u \in H^{1}\left(\mathbb{R}^{3}\right)\{\{0\}} \max _{t>0} E\left(t^{2} u_{t}\right) .
$$

Lemma 2.8. Assume that (V1), (V2) hold. Then

(i) There exists $\delta>0$ such that $\|u\| \geq \delta, \forall u \in \mathcal{M}$;

(ii) $m=\inf _{\mathcal{M}} E(u)>0$.

Proof. (i) Since $J(u)=0, \forall u \in \mathcal{M}$, by (1.8), (2.1) and Sobolev embedding theorem, one has

$$
\begin{aligned}
\frac{\min \left\{3, \rho_{2}\right\}}{2}\|u\|^{2} & \leq \frac{3}{2}\|\nabla u\|_{2}^{2}+\frac{1}{2} \int_{\mathbb{R}^{3}}[V(x)-\nabla V(x) \cdot x] u^{2} \mathrm{~d} x+\frac{3 \lambda}{4} \int_{\mathbb{R}^{3}} \phi_{u}(x) u^{2} \mathrm{~d} x \\
& =\frac{3}{2} \int_{\mathbb{R}^{3}}\left(I_{\alpha} *|u|^{3+\alpha}\right)|u|^{3+\alpha} \mathrm{d} x \\
& \leq C_{1}\|u\|^{2(3+\alpha)},
\end{aligned}
$$

which implies

$$
\|u\| \geq \delta:=\min \left\{1,\left(\frac{\min \left\{3, \rho_{2}\right\}}{2 C_{1}}\right)^{\frac{1}{4+2 \alpha}}\right\}, \quad \forall u \in \mathcal{M} .
$$

(ii) Let $\left\{u_{n}\right\} \subset \mathcal{M}$ be such that $E\left(u_{n}\right) \rightarrow m$. Cases: (1) $\inf _{n \in \mathbb{N}}\left\|u_{n}\right\|_{2}>0$; (2) $\inf _{n \in \mathbb{N}}\left\|u_{n}\right\|_{2}=0$ exist.

Case (1). $\inf _{n \in \mathbb{N}}\left\|u_{n}\right\|_{2}:=\delta_{0}>0$. By (2.4), one has

$$
m=E\left(u_{n}\right)=E\left(u_{n}\right)-\frac{1}{3} J\left(u_{n}\right) \geq \frac{\rho_{1}}{6} \delta .
$$

Case (2). $\inf _{n \in \mathbb{N}}\left\|u_{n}\right\|_{2}=0$. From (2.19), we have

$$
\left\|u_{n}\right\|_{2} \rightarrow 0, \quad\left\|\nabla u_{n}\right\|_{2} \geq \frac{1}{2} \delta .
$$

By Hardy-Littlewood-Sobolev inequality, one has

$$
\int_{\mathbb{R}^{3}}\left(I_{\alpha} *\left|u_{n}\right|^{3+\alpha}\right)\left|u_{n}\right|^{3+\alpha} \mathrm{d} x \leq C_{2}\left\|\nabla u_{n}\right\|_{2}^{2(3+\alpha)} .
$$

Let $t_{n}=\left(\frac{1}{4 C_{2}}\right)^{-\frac{1}{3(2+\alpha)}}\left\|\nabla u_{n}\right\|_{2}^{-\frac{2}{3}}$, then (2.21) implies that $t_{n}$ is bounded. Since $J\left(u_{n}\right)=0$, it follows from (2.15) and (2.22) that

$$
\begin{aligned}
m= & E\left(u_{n}\right) \geq E\left(t_{n}^{2}\left(u_{n}\right)_{t_{n}}\right) \\
= & \frac{t_{n}^{3}}{2} \|\left.\nabla u_{n}\right|_{2} ^{2}+\frac{t_{n}}{2} \int_{\mathbb{R}^{3}} V\left(t^{-1} x\right) u_{n}^{2} \mathrm{~d} x+\frac{\lambda t^{3}}{4} \int_{\mathbb{R}^{3}} \phi_{u_{n}}(x) u_{n}^{2} \mathrm{~d} x \\
& -\frac{t_{n}^{9+3 \alpha}}{2(3+\alpha)} \int_{\mathbb{R}^{3}}\left(I_{\alpha} *\left|u_{n}\right|^{3+\alpha}\right)\left|u_{n}\right|^{3+\alpha} \mathrm{d} x \\
\geq & \frac{t_{n}^{3}}{2}\left\|\nabla u_{n}\right\|_{2}^{2}-C_{2} t_{n}^{9+3 \alpha}\left\|\nabla u_{n}\right\|_{2}^{2(3+\alpha)} \\
= & \frac{1}{2} t_{n}^{3}\left\|\nabla u_{n}\right\|_{2}^{2}\left[1-2 C_{2}\left(t_{n}^{3}\left\|\nabla u_{n}\right\|_{2}^{2}\right)^{2+\alpha}\right]>0 .
\end{aligned}
$$


Cases (1) and (2) show that (ii) holds.

Lemma 2.9. Assume that (V1) and(V2) hold. Then $m^{\infty}:=\inf _{\mathcal{M}^{\infty}} \Phi^{\infty} \geq m$.

Proof. In view of Lemma 2.1 and Corollary 2.3 , we have $\mathcal{M} \neq \varnothing$. By contradiction, we assume that $m>m^{\infty}$. Let $\rho:=m-m^{\infty}$. Then there exists $u_{\varepsilon}^{\infty}$ such that

$$
u_{\rho}^{\infty} \in \mathcal{M}^{\infty} \text { and } m^{\infty}+\frac{\rho}{2}>E^{\infty}\left(u_{\rho}^{\infty}\right) .
$$

In view of Lemma 2.5, there exists $t_{\rho}>0$ such that $\left(u_{\rho}\right)_{t_{\rho}} \in \mathcal{M}$. Hence, joining with (V1), (V2), (1.10), (2.5), (2.11) and (2.24), we have

$$
m^{\infty}+\frac{\rho}{2}>E^{\infty}\left(u_{\rho}^{\infty}\right) \geq E^{\infty}\left(\left(u_{\rho}^{\infty}\right)_{t_{\rho}}\right) \geq E\left(\left(u_{\rho}^{\infty}\right)_{t_{\rho}}\right) \geq m .
$$

This is a contradiction. Therefore, the conclusion of Lemma 2.11 is true.

Lemmma 2.10. ([11]: Lemma 2.12) Assume that (V1) and(V2) hold. If $u_{n} \rightarrow \bar{u}$ in $H^{1}\left(\mathbb{R}^{3}\right)$, then along a subsequence,

$$
\begin{gathered}
E\left(u_{n}\right)=E(\bar{u})+E\left(u_{n}-\bar{u}\right)+o(1), \quad J\left(u_{n}\right)=J(\bar{u})+J\left(u_{n}-\bar{u}\right)+o(1) \\
E^{\prime}\left(u_{n}\right)=E^{\prime}(\bar{u})+E^{\prime}\left(u_{n}-\bar{u}\right)+o(1) \\
\left\langle E^{\prime}\left(u_{n}\right), u_{n}\right\rangle=\left\langle E^{\prime}(\bar{u}), \bar{u}\right\rangle+\left\langle E^{\prime}\left(u_{n}-\bar{u}\right), u_{n}-\bar{u}\right\rangle+o(1)
\end{gathered}
$$

Lemma 2.11. Assume that (V1), (V2) hold. Then $m$ is achieved.

Proof. In view of Lemmas 2.5 and 2.8, we have $\mathcal{M} \neq \varnothing$ and $m>0$. Let $\left\{u_{n}\right\} \subset \mathcal{M}$ be such that $E\left(u_{n}\right) \rightarrow m$. Then it follows from (1.10), (2.1) and (2.27) that

$$
m=E\left(u_{n}\right)=E\left(u_{n}\right)-\frac{1}{3} J\left(u_{n}\right) \geq \frac{\rho_{1}}{3}\left\|u_{n}\right\|_{2}^{2} .
$$

It indicates that $\left\{\left\|u_{n}\right\|_{2}\right\}$ is bounded. Next, we will verify that $\left\{\|\nabla u\|_{2}\right\}$ is also bounded. From (V1), (1.10), (2.1), (2.13), (2.29) and the Soblev embbeding inequality, we derive

$$
\begin{aligned}
E\left(u_{n}\right)= & E\left(u_{n}\right)-\frac{1}{3(3+\alpha)} J\left(u_{n}\right) \\
= & \frac{2+\alpha}{2(3+\alpha)}\left\|\nabla u_{n}\right\|_{2}^{2}+\frac{1}{2} \int_{\mathbb{R}^{3}} V(x) u_{n}^{2} \mathrm{~d} x \\
& +\frac{1}{6(3+\alpha)} \int_{\mathbb{R}^{3}}[V(x)-\nabla V(x) \cdot x] u_{n}^{2} \mathrm{~d} x \\
& +\frac{(2+\alpha) \lambda}{4(3+\alpha)} \int_{\mathbb{R}^{3}}\left(I_{\alpha} *|u|^{3+\alpha}\right)|u|^{3+\alpha} \mathrm{d} x \\
\geq & \frac{2+\alpha}{2(3+\alpha)}\left\|u_{n}\right\|^{2}+\frac{\rho_{2}-6-2 \alpha}{6(3+\alpha)}\left\|u_{n}\right\|_{2}^{2} .
\end{aligned}
$$

Together with (2.29), (2.30) implies that $\left\{u_{n}\right\}$ is bounded in $H^{1}\left(\mathbb{R}^{3}\right)$. Passing to a subsequence, we can get $u_{n} \rightarrow \bar{u}$ in $H^{1}\left(\mathbb{R}^{3}\right)$. Then $u_{n} \rightarrow \bar{u}$ in $L_{\text {loc }}^{s}\left(\mathbb{R}^{3}\right)$ for $2 \leq s<6$ and $u_{n} \rightarrow \bar{u}$ a.e. in $\mathbb{R}^{3}$. For $\bar{u}$, there are two cases: (1) $\bar{u}=0$ and (2) $\bar{u} \neq 0$. 
Case (1). $\bar{u}=0$, i.e. $u_{n} \rightarrow 0$ in $H^{1}\left(\mathbb{R}^{3}\right)$. Then $u_{n} \rightarrow 0$ in $L_{\text {loc }}^{s}\left(\mathbb{R}^{3}\right)$ for $2 \leq s<2^{*}$ and $u_{n} \rightarrow 0$ a.e. in $\mathbb{R}^{3}$. Using (V1) and (V2), it is easy to prove that

$$
\lim _{n \rightarrow \infty} \int_{\mathbb{R}^{3}}\left[V_{\infty}-V(x)\right] u_{n}^{2} \mathrm{~d} x=\lim _{n \rightarrow \infty} \int_{\mathbb{R}^{3}} \nabla V(x) \cdot x u_{n}^{2} \mathrm{~d} x=0 .
$$

From (1.10), (2.1), (2.8), (2.10) and (2.31), one has

$$
\tau^{\infty}\left(u_{n}\right) \rightarrow m, \quad J^{\infty}\left(u_{n}\right) \rightarrow 0 .
$$

By (1.8), (2.1) and Lemma 2.8 (i), we have

$$
\begin{aligned}
\frac{\min \left\{3, \rho_{2}\right\}}{2} \delta^{2} & \leq \frac{3}{2}\left\|\nabla u_{n}\right\|_{2}^{2}+\frac{1}{2} \int_{\mathbb{R}^{3}}[V(x)-\nabla V(x) \cdot x] u_{n}^{2} \mathrm{~d} x+\frac{3 \lambda}{4} \int_{\mathbb{R}^{3}} \phi_{u} u_{n} \mathrm{~d} x \\
& =\frac{3}{2} \int_{\mathbb{R}^{3}}\left(I_{\alpha} *\left|u_{n}\right|^{3+\alpha}\right)\left|u_{n}\right|^{3+\alpha} \mathrm{d} x \\
& \leq C_{3}\left\|u_{n}\right\|^{2(3+\alpha)} .
\end{aligned}
$$

According to (2.33) and Lion's concentration compactness priciple ([21]: Lemma 1.21), we can prove that there exist $\delta>0$ and $y_{n} \in \mathbb{R}^{3}$ such that $\int_{B_{1}\left(y_{n}\right)}\left|u_{n}\right|^{2} \mathrm{~d} x>\delta$. Let $\hat{u}_{n}(x)=u_{n}\left(x+y_{n}\right)$. Then we have $\left\|\hat{u}_{n}\right\|=\left\|u_{n}\right\|$ and

$$
J^{\infty}\left(\hat{u}_{n}\right)=o(1), \quad E^{\infty}\left(\hat{u}_{n}\right) \rightarrow m, \quad \int_{B_{1}(0)}\left|\hat{u}_{n}\right| \mathrm{d} x>\delta .
$$

Hence, there exists $\hat{u} \in H^{1}\left(\mathbb{R}^{3}\right) \backslash\{0\}$ such that, passing to a subsequence,

$$
\left\{\begin{array}{l}
\hat{u}_{n} \rightarrow \hat{u} \text {, in } H^{1}\left(\mathbb{R}^{3}\right) ; \\
\hat{u}_{n} \rightarrow \hat{u} \text {, in } L_{\text {loc }}^{s}\left(\mathbb{R}^{3}\right), \forall s \in[1,6) ; \\
\hat{u}_{n} \rightarrow \hat{u} \text {, a.e. on } \mathbb{R}^{3} .
\end{array}\right.
$$

Let $w_{n}=\hat{u}_{n}-\hat{u}$. Then (2.35) and Lemma 2.10 yield

$$
E^{\infty}\left(\hat{u}_{n}\right)=E^{\infty}(\hat{u})+E^{\infty}\left(w_{n}\right)+o(1), \quad J^{\infty}(\hat{u})=J^{\infty}(\hat{u})+J^{\infty}\left(w_{n}\right)+o(1) .
$$

We set

$$
E^{\infty}(u)=E^{\infty}(u)-\frac{1}{3} J^{\infty}(u)=\frac{V_{\infty}}{3}\|u\|_{2}^{2}+\left(\frac{1}{6}-\frac{1}{6+2 \alpha}\right) \int_{\mathbb{R}^{3}}\left(I_{\alpha} *|u|^{\frac{\alpha}{3}+1}\right)|u|^{\frac{\alpha}{3}+1} \mathrm{~d} x(
$$

From (2.8), (2.10), (2.24), (2.36) and (2.37), one has

$$
E^{\infty}\left(w_{n}\right)=m-E^{\infty}(\hat{u})+o(1), \quad J^{\infty}\left(w_{n}\right)=-J^{\infty}(\hat{u})+o(1) .
$$

If there exists a subsequence $\left\{w_{n_{i}}\right\}$ of $\left\{w_{n}\right\}$ such that $w_{n_{i}}=0$, then we have

$$
E^{\infty}(\hat{u})=m, \quad J^{\infty}(\hat{u})=0 .
$$

Next, we consider that $w_{n} \neq 0$. We claim that $J^{\infty}(\hat{u}) \leq 0$. By contradiction, when $J^{\infty}(\hat{u})>0$, that is (2.38) implies $J^{\infty}\left(w_{n}\right)<0$ for large $n$. In view of Corollary 2.6, there exists $t_{n}>0$ such that $t_{n}^{2}\left(w_{n}\right)_{t_{n}} \in \mathcal{M}^{\infty}$ for large $n$. From (2.8), (2.10), (2.11), (2.38) and Lemma 2.9, one has

$$
\begin{aligned}
m-E^{\infty}(\hat{u})+o(1) & =E^{\infty}\left(w_{n}\right)=E^{\infty}\left(w_{n}\right)-\frac{1}{3} J^{\infty}\left(w_{n}\right) \\
& \geq E^{\infty}\left(t_{n}^{2}\left(w_{n}\right)_{t_{n}}\right)-\frac{t_{n}^{3}}{3} J^{\infty}\left(w_{n}\right)+\frac{\left(1-t_{n}\right)^{2}\left(2+t_{n}\right) V_{\infty}}{6}\left\|w_{n}\right\|_{2}^{2}
\end{aligned}
$$




$$
\geq m-\frac{t_{n}^{3}}{3} J^{\infty}\left(w_{n}\right) \geq m
$$

Since $\Phi^{\infty}(\hat{u})>0$, the above result is impossible, this shows that $J^{\infty}(\hat{u}) \leq 0$. In view of Lemma 2.1, there exists $t_{\infty}>0$ such that $t_{\infty}^{2} \hat{u}_{t_{\infty}} \in \mathcal{M}^{\infty}$. From (2.8), (2.10), (2.11), (2.32), (2.34), (2.37) Fatou's lemma and Lemma 2.9, one has

$$
\begin{aligned}
m & =\lim _{n \rightarrow \infty}\left[E^{\infty}\left(\hat{u}_{n}\right)-\frac{1}{3} J^{\infty}\left(\hat{u}_{n}\right)\right] \\
& =\lim _{n \rightarrow \infty} E\left(\hat{u}_{n}\right) \geq E(\hat{u})=E^{\infty}(\hat{u})-\frac{1}{3} J^{\infty}(\hat{u}) \\
& \geq E^{\infty}\left(t_{\infty}^{2} \hat{u}_{t_{\infty}}\right)-\frac{t_{\infty}^{3}}{3} J(\hat{u})+\frac{\left(1-t_{\infty}\right)^{2}\left(2+t_{\infty}\right)}{6} \int_{\mathbb{R}^{3}} V_{\infty} \hat{u}^{2} \mathrm{~d} x \\
& \geq m-\frac{t_{\infty}^{3}}{3} J^{\infty}(\hat{u})+\frac{\left(1-t_{\infty}\right)^{2}\left(2+t_{\infty}\right) V_{\infty}}{6}\|\hat{u}\|_{2}^{2} \geq m,
\end{aligned}
$$

which implies (2.39) holds also. In view of Lemma 2.3, there exists $\hat{t}>0$ such that $\hat{t}^{2} \hat{u}_{\hat{t}} \in \mathcal{M}$, moreover, it follows from (V1), (1.10), (2.8), (2.39) and Corollary 2.3 that

$$
m \leq E\left(\hat{t}^{2} \hat{u}_{\hat{t}}\right) \leq E^{\infty}\left(\hat{t}^{2} \hat{u}_{\hat{t}}\right) \leq E^{\infty}(\hat{u})=m .
$$

Case (ii). $\bar{u} \neq 0$. In this case, the proof is similar to (2.39), by using $E$ and $J$ instead of $E^{\infty}$ and $J^{\infty}$, we can deduce that $E(\bar{u})=m$ and $J(\bar{u})=0$.

Similar to the [22] and [23], we can obtain the following conclusion.

Lemma 2.12. Assume that (V1), (V2) hold. If $\bar{u} \in \mathcal{M}$ and $E(\bar{u})=m$, then $\bar{u}$ is a critical point of $E$.

Proof. From (2.1) and Lemma 2.5, there exist $T_{1} \in(0,1)$ and $T_{2} \in(1, \infty)$ such that

$$
E\left(T_{1}^{2} \tilde{u}_{T_{1}}\right)>0, \quad E\left(T_{2}^{2} \tilde{u}_{T_{2}}\right)<0 .
$$

From (2.2) and (2.4), we have

$$
E\left(t^{2} \tilde{u}_{t}\right) \leq E(\tilde{u})-\frac{1}{6} \int_{\mathbb{R}^{3}} f(x, t) \tilde{u}^{2} \mathrm{~d} x<m, \quad \forall t \in(0,1) \cup(1, \infty) .
$$

and (2.44) implies

$$
E:=\max \left\{E\left(T_{1}^{2} \tilde{u}_{T_{1}}\right), E\left(T_{1}^{2} \tilde{u}_{T_{2}}\right)\right\}<m .
$$

The next proof steps are routine. Similar to [22], we can verify Lemma 2.12 by using (2.43) and (2.44) instead of ([22]: (2.34) and (2.35)).

Proof of Theorem 1.1. In view of Lemmas 2.9 and 2.10, there exists $\bar{u} \in \mathcal{M}$ such that

$$
E(\bar{u})=m=\inf _{u \in H^{1}\left(\mathbb{R}^{3}\right)\{\{0\}} \max _{t>0} E\left(t^{2} u_{t}\right), \quad E^{\prime}(\bar{u})=0 .
$$

This shows that $\bar{u}$ is a ground state solution of (1.4).

\section{Conclusion}

Although one can establish a (PS) sequence in a nonstandard way, it is not easy 
to prove its boundness because of the convolution term $\left(I_{\alpha} *|u|^{3+\alpha}\right)|u|^{1+\alpha} u$ and lack of Ambrosetti-Rabinowitz condition of Choquard type. To overcome this difficulty, we introduce an auxiliary function. Firstly, we proved that there exists a unique $t_{u}>0$ such that $t_{u}^{2} u_{t_{u}} \in \mathcal{M}$. What's more, we find out the minima of the energy functional. Next, we get that $m$ is achieved, that is the energy value of the minima of the energy functional is achieved by Mountain Pass Theorem. Finally, we proved that the limit of the (PS) sequence, that is $\bar{u}$, is the critical point of $E$. It is obvious that for the Schrödinger-Poisson system with upper critical exponential convolution term, its ground state solution also exists. We hope the result can be widely used in Schrödinger-Poisson systems.

\section{Acknowledgements}

The authors would like to thank the referees for their useful suggestions which have significantly improved the paper.

\section{Funding}

This work is supported by the National Natural Science Foundation of China (No. 11961014) and Guangxi Natural Science Foundation (2021GXNSFAA196040).

\section{Authors' Contributions}

The authors declare that the study was realized in collaboration with the same responsibility. All authors read and approved the final manuscript.

\section{Conflicts of Interest}

The authors declare that they have no competing interests.

\section{References}

[1] Benci, V. and Donato, F. (1998) An Eigenvalue Problem for the Schrödinger-Maxwell Equations. Topological Methods in Nonlinear Analysis, 11, 283-293. https://doi.org/10.12775/TMNA.1998.019

[2] D’Aprile, T. and Mugnai, D. (2004) Solitary Waves for Nonlinear Klein-GordonMaxwell and Schrödinger-Maxwell Equations. Proceedings of the Royal Society of Edinburgh Section A, 134, 893-906. https://doi.org/10.1017/S030821050000353X

[3] Khoutir, S. (2021) Least Energy Sign-Changing Solutions for Super-Quadratic Schrödinger-Poisson Systems in $\mathbb{R}^{3}$. Journal of Applied Analysis \& Computation, 11, 1520-1543.

[4] Chen, S.T., Shi, J.P. and Tang, X.H. (2019) Ground State Solutions of Nehari-Pohožaev Type for the Planar Schrödinger-Poisson System with General Nonlinearity. Discrete \& Continuous Dynamical Systems, 39, 5867-5889. https://doi.org/10.3934/dcds.2019257

[5] Alves, C.O., Souto, M.A.S. and Soares, S.H.M. (2017) A Sign-Changing Solution for the Schrödinger-Poisson Equation in $\mathbb{R}^{3}$. Rocky Mountain Journal of Mathematics, 47, 1-25. https://doi.org/10.1216/RMJ-2017-47-1-1

[6] Shuai, W. and Wang, Q. (2015) Existence and Asymptotic Behavior of Sign-Changing Solutions for the Nonlinear Schrödinger-Poisson System in $\mathbb{R}^{3}$. Zeitschrift für an- 
gewandte Mathematik und Physik, 66, 3267-3282.

https://doi.org/10.1007/s00033-015-0571-5

[7] Liang, Z., Xu, J. and Zhu, X. (2016) Revisit to Sign-Changing Solutions for the Nonlinear Schrödinger-Poisson System in $\mathbb{R}^{3}$. Journal of Mathematical Analysis and Applications, 435, 783-799. https://doi.org/10.1016/j.jmaa.2015.10.076

[8] Chen, S.T. and Tang, X.H. (2020) Berestycki-Lions Conditions on Ground State Solutions for a Nonlinear Schrödinger Equation with Variable Potentials. Advances in Nonlinear Analysis, 9, 496-515. https://doi.org/10.1515/anona-2020-0011

[9] Bartsch, T., Liu, Z.L. and Weth, T. (2004) Sign Changing Solutions of Superlinear Schrödinger Equations. Communications in Partial Differential Equations, 29, 25-42. https://doi.org/10.1081/PDE-120028842

[10] Guo, H. and Wu, D. (2020) Nodal Solutions for the Schrödinger-Poisson Equations with Convolution Terms. Nonlinear Analysis, 196, Article ID: 111781. https://doi.org/10.1016/j.na.2020.111781

[11] Chen, S.T. and Tang, X.H. (2019) Ground State Solutions of Schrödinger-Poisson Systems with Variable Potential and Convolution Nonlinearity. Journal of Mathematical Analysis and Applications, 473, 87-111. https://doi.org/10.1016/j.jmaa.2018.12.037

[12] Lieb, E.H. and Loss, M. (1997) Analysis Graduate Studies in Mathematics. American Mathematical Society, Providence.

[13] Wang, Z. and Zhou, H.S. (2021) Ground State for Nonlinear Schrödinger Equation with Sign-Changing and Vanishing Potential. Journal of Mathematical Physics, 52, Article ID: 113704. https://doi.org/10.1063/1.3663434

[14] Liu, X. and Huang, Y. (2009) Sign-Changing Solutions for a Class of Nonlinear Schrödinger Equations. Bulletin of the Australian Mathematical Society, 80, 294-305. https://doi.org/10.1017/S0004972709000288

[15] Chen, J. (2006) Multiple Positive and Sign-Changing Solutions for a Singular Schrödinger Equation with Critical Growth. Nonlinear Analysis: Theory, Methods \& Applications, 64, 381-400. https://doi.org/10.1016/j.na.2005.03.102

[16] Schaftingen, J.V. and Xia, J.K. (2018) Ground States for a Local Nonlinear Perturbation of the Choquard Equations with Lower Critical Exponent. Journal of Mathematical Analysis and Applications, 464, 1184-1202.

https://doi.org/10.1016/j.jmaa.2018.04.047

[17] Tang, X.H. and Chen, S.T. (2020) Singularly Perturbed Choquard Equations with Nonlinearity Satisfying Berestycki-Lions Assumptions. Advances in Nonlinear Analysis, 9, 413-437. https://doi.org/10.1515/anona-2020-0007

[18] Alves, C.O., Gao, F.S., Squassinac, M. and Yang, M.B. (2017) Singularly Perturbed Critical Choquard Equations. Journal of Differential Equations, 263, 3943-3988. https://doi.org/10.1016/j.jde.2017.05.009

[19] Seok, J. (2018) Nonlinear Choquard Equations: Doubly Critical Case. Applied Mathematics Letters, 76, 148-156. https://doi.org/10.1016/j.aml.2017.08.016

[20] Shen, L.J. (2021) Ground State Solutions for Planar Schrödinger-Poisson System Involving Subcritical and Critical Exponential Growth with Convolution Nonlinearity. Journal of Mathematical Analysis and Applications, 495, Article ID: 124662. https://doi.org/10.1016/j.jmaa.2020.124662

[21] Willem, M. and Theorems, M. (1996) Progress in Nonlinear Differential Equations and Their Applications. Vol. 24, Birkhöuser Boston Inc., Boston.

[22] Chen, S.T. and Tang, X.H. (2018) Ground State Solutions for Generalized Quasili- 
near Schrödinger Equations with Variable Potentials and Berestycki-Lions Nonlinearities. Journal of Mathematical Physics, 59, Article ID: 081508.

https://doi.org/10.1063/1.5036570

[23] Wang, X.P. and Liao, F.F. (2020) Ground State Solutions for a Choquard Equation with Lower Critical Exponent and Local Nonlinear Perturbation. Nonlinear Analysis, 196, Article ID: 111831. https://doi.org/10.1016/j.na.2020.111831 